\title{
Case Report \\ Effect of Bakri Balloon on Managing Postpartum Hemorrhage: A Case Series
}

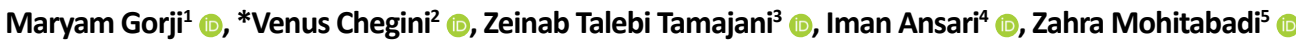

1. Velayat Hospital, Qazvin University of Medical Sciences, Qazvin, Iran.

2. Department of Obestetrics and Gynecology, School of Medicine, Qazvin University of Medical Sciences, Qazvin, Iran.

3. Department of Midwifery, School of Midwifery, Alborz University of Medical Sciences, Karaj, Iran.

4. Medical Students Research Committee, School of Medicine, Shahed University, Tehran, Iran.

5. Kosar Hospital, Qazvin University of Medical Sciences, Qazvin, Iran

\begin{tabular}{|c|c|}
\hline $\begin{array}{l}\text { Use your device to scan } \\
\text { and read the article online }\end{array}$ & \\
\hline 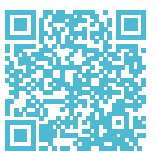 & $\begin{array}{l}\text { Citation Gorji M, Chegini V, Talebi Tamajani Z, Ansari I, Mohitabadi Z. Effect of Bakri Balloon on Managing Postpartum Hemorrhage: } \\
\text { A Case Series. The Journal of Qazvin University of Medical Sciences. 2019; 23(3):278-285. https://doi.org/10.32598/JQUMS.23.3.278 } \\
\text { doil" https://doi.org/10.32598/JQUMS.23.3.278 }\end{array}$ \\
\hline
\end{tabular}

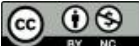

Received: 30 Jun 2018 Accepted: 24 Nov 2018 Available Online: 01 Aug 2019 Keywords: Postpartum hemorrhage, Uterine balloon tamponade, Uterine atony

\section{ABSTRACT}

Postpartum hemorrhage (PPH) is among the most significant causes of maternal death worldwide. The time of diagnosing and the proper management of hemorrhage are essential in preventing maternal mortality. The current study aimed to evaluate the effect of Bakri balloon on the management of PPH. Bakri balloon was effective in controlling PPH of $92.3 \%$ of patients. The most frequent indications for using Bakri balloon was uterine atony (46.1\%). Furthermore, Bakri balloon is among the simplest and low invasive methods that can be used to control PPH before invasion methods, like hysterectomy.

\section{Extended Abstract}

\section{Introduction}

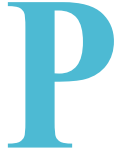

ostpartum hemorrhage (PPH) is the most common cause of maternal mortality worldwide. There are two types of PPH; 1. Primary PPH that appears as bleeding during the first 24 hours after delivery; and 2. Secondary PPH, developed 24 hours and up to 6 weeks after postpartum [1]. PPH control depends on its cause. PPH can be associated with severe and widespread complications, including hypovolemic shock, renal dysfunction and coagulation problems [2]. The first-line treatment method for this condition is conservative management and the use of uterotonics, including oxytocin and prostaglandin. The second-line option includes the use of uterine packing, intrauterine balloon tamponade, external compression with uterine sutures, and uterine artery embolization [2]. If none of these options was effective, hysterectomy is required. Hysterectomy may be associated with more blood loss and many short- and long-term complications, such as injury to other organs, infection and impaired wound healing, fertility loss, prolonged healing period, and so on [3].

Balloon tamponade has been suggested as an alternative method, which can significantly control PPH [4]. The efficacy of Bakri balloon tamponade has also been reported in

\section{* Corresponding Author:}

Venus Chegini

Address: Department of Obstetrics and Gynecology, School of Medicine, Qazvin University of Medical Sciences, Qazvin, Iran.

Tel: +98 (28) 33236374

E-Mail: venus_chegini@yahoo.com 
various studies as a new tool to control PPH with a high success rate [5]. The balloon is easily inserted and quickly removed and its special shape and silicone material prevents the balloon from adhering to the inner layer of the uterus, causes sufficient pressure to tampon the uterus, and extensively controls bleeding. In addition, the exact rate of simultaneous blood loss is assessed by its drainage port [6]. Although the balloon may seem useful in controlling PPH, studies are limited regarding the Bakri balloon and its effect on reducing PPH, particularly in Iran. Therefore, this study aimed to evaluate the effect of Bakri balloon on controlling PPH among hospitalized patients.

\section{Materials and Methods}

This case series study was conducted from October 2015 to March 2017 in Kosar Hospital in Qazvin City, Iran. The study participants were patients whose PPH failed to respond to pharmacological treatment (e.g. oxytocin, methylergonovine, and misoprostol); thus, Bakri balloon was used to control their PPH.

Information on age, occupation, residence place, the number of births, gestational age, hospitalization cause, delivery method, serum hemoglobin level before and after delivery, the duration of stay, intensive care unit (ICU) admission, blood transfusion, indication and the duration of using Bakri balloon, and Bakri balloon effect on bleeding control were collected from the patients' records.

\section{Results}

The collected results reported that the most common cause of hospitalization was vaginal bleeding (46.2\%). Eleven (84.7\%) patients had Caesarean-section (C-section); 7 of which were due to previous $\mathrm{C}$-section, one due to twin pregnancy, and 3 because of a drop in the fetal heart rate. Twelve $(92.3 \%)$ patients were admitted to ICU for at least 12 hours, and $11(84.7 \%)$ patients required blood transfusions and blood products. The Mean \pm SD serum hemoglobin concentration in patients before and after delivery were $12.4 \pm 1.8$ and $9.2 \pm 1.1 \mathrm{~g} / \mathrm{dL}$, respectively. The most frequent cause of Bakri balloon use was uterine atony (46.1\%), followed by Placenta Accreta (30.8\%), and Placenta Percreta (23.1\%). The Mean \pm SD duration of stay for Bakri balloon in the uterus was $27 \pm 18 \mathrm{~h}$. The use of Bakri balloon was effective in controlling PPH in $12(92.3 \%)$ patients, including all those with vaginal deliveries and $90.9 \%$ of those with C-section. In one case, where PPH failed to respond to medical procedures and the use of Bakri balloon due to Placenta Percreta, hysterectomy was performed.

\section{Conclusion}

The present study revealed the Bakri balloon use was more effective in controlling PPH in 12 (92.3\%) patients, including all women with vaginal delivery and $90.9 \%$ of women with $\mathrm{C}$-section, compared to conventional medication use. In thea study by Vitthala et al. on 15 patients, the overall success rate of the Bakri balloon use in controlling PPH was reported as $80 \%$ [7]. In their study, like the present study, PPH was $100 \%$ controlled in women with vaginal delivery; whereas, Bakri balloon use was effective in only $57 \%$ of women with C-section. Georgiou and Wang et al. reported the overall success rate of the Bakri balloon in PPH control as $91.5 \%$ and $91.6 \%$, respectively $[8,9]$. This information is close to the results of our study. According to Aibar et al., the overall rate of success of Bakri balloon use in PPH control was equal to $87.5 \%$, and for patients with vaginal delivery, it was $100 \%$ [10]. The most common reason for the use of Bakri balloon in their study was uterine atony (33.3\%).

Consistent with their study, our results suggested that Bakri balloon use was quite effective in controlling PPH in patients with vaginal delivery. Furthermore, uterine atony with $46.1 \%$ was the most common reason for the use of Bakri balloon. Overall, it seems that the Bakri balloon is one of the simple, feasibly-used, and low-invasive procedures with no serious complications; thus, it can be used in PPH control before invasive procedures, like s hysterectomy. It also reduces the bleeding, the need for blood transfusions, and hospital stay duration.

One of the limitations of the present study was the small sample size and the limited number of variables included in the patients' record. Therefore, to be able to widely use the Bakri balloon in controlling PPH, it is necessary to conduct research studies with a larger statistical population and investigate more variables comparable with other methods in PPH control.

\section{Ethical Considerations}

\section{Compliance with ethical guidelines}

This study was approved by the Ethics Committee of Qazvin University of Medical Sciences (Code: IR.QUMS. REC.1397.051).

\section{Funding}

This research did not receive any specific grant from funding agencies in the public, commercial, or not-forprofit sectors. 


\section{Authors' contributions}

Design, data collection and drafting: Maryam Gorji, Venus Chegini, and Zeinab Talebi Tamajani; Contributed to data analysis and interpretation and content correction: Iman Ansari and Zahra Mohitabadi; Endorse the final version and agree on the completeness and accuracy of this research: All authors.

Conflicts of interest

There were no conflicts of interest in this study.

Acknowledgements

From the sincere cooperation of Dr. Maleki, president of the Kowsar Medical Center of Qazvin and Dr. Sara Esmaeilizadeh, a member of faculty of Alborz University of Medical Sciences thanks and appreciate. 


\title{
تأثير بالون بكرى در كتترل خونريزى بعد از زايمان: تَزارش موارد
}

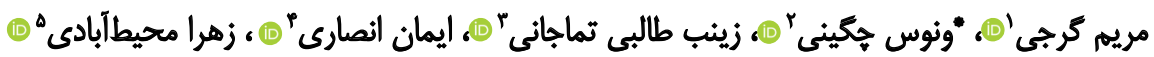

ا-بيمارستان ولايت، دانشكاه علوم يزشكى قزوين، قزوين، ايران.

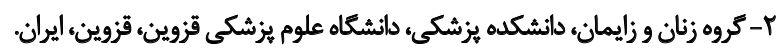

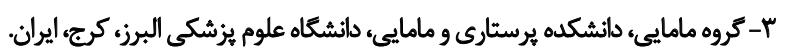

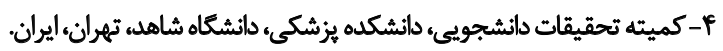

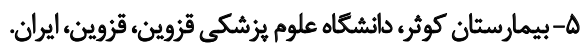

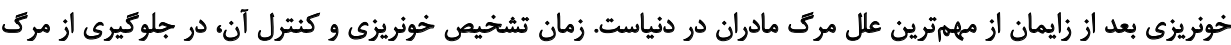

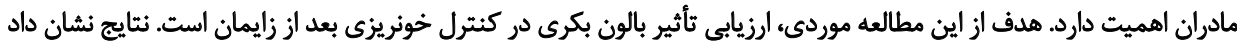

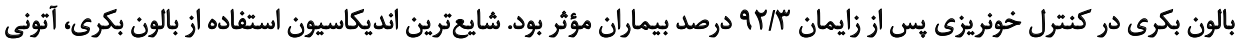

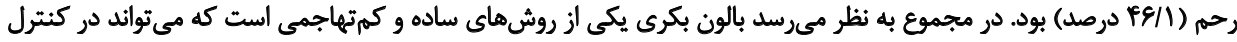

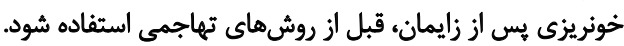

تاريخ دريافت: 9 تير

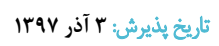

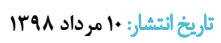

\section{:}

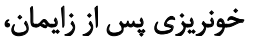
بالون تاميوناد رحمه، آتونى ران تاميوناد
كليه و مشكلات انعقادى همراه باشد [ب]. در سراسر جهان در هر

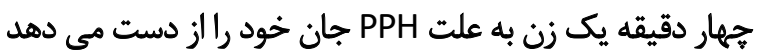

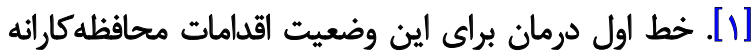

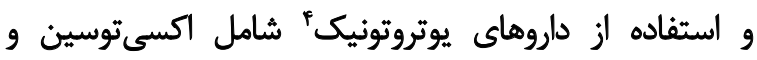

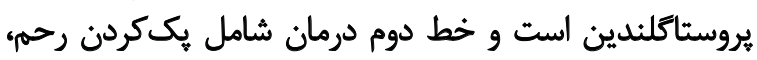

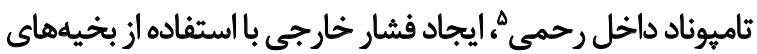

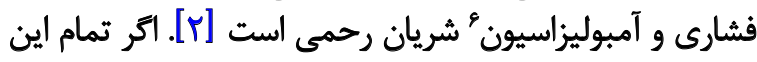

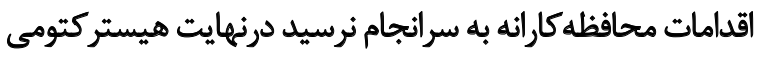

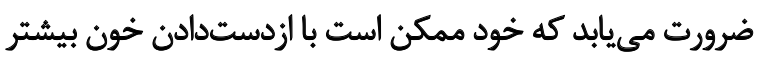

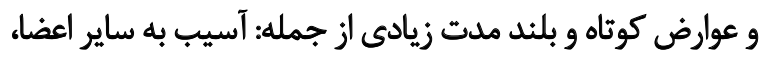

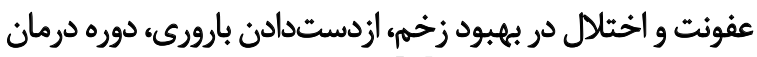
طولانى و غيره همراه باشد [بآ]

سازمان جهانى بهداشت، فدراسيون بينالمللى زنان و مامايى'؛

\section{Uterotonics}

5. Intrauterine bballoon tamponade

6. Embolization

7. International federation of gynecology and obstetrics (FIGO)
Lâa

خونريزى بس از زايمان' جشمثيرترين علت مركى مادران در

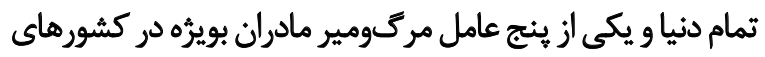

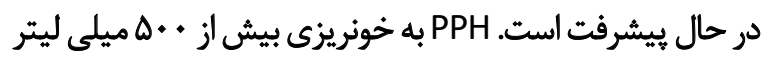

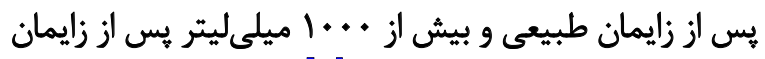

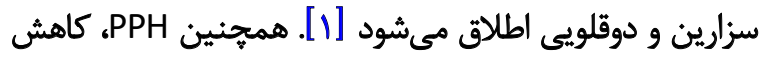

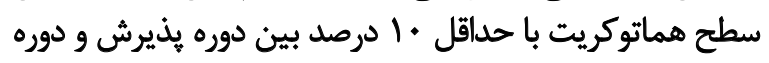

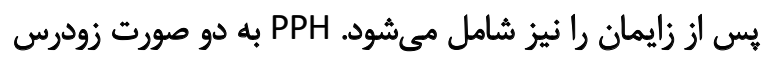

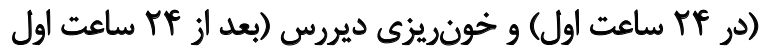

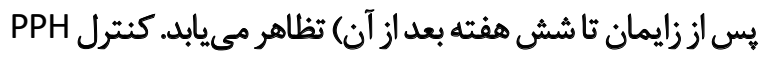

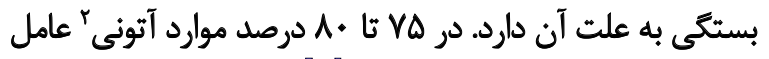

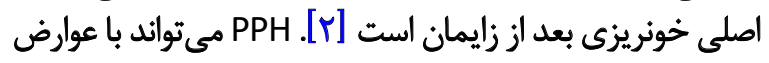

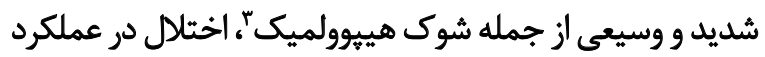

1. Postpartum hemorrhage (PPH)

2. Atony

3. Hypovolemic shok

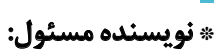
ونوس جزكينى

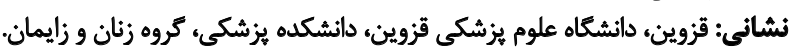
تلفن: رايانامه: vلفن: venus_chegini@yahoo.com 


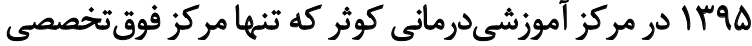

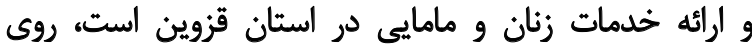

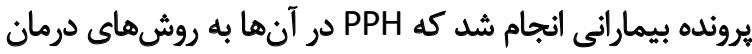

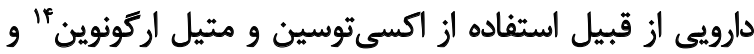

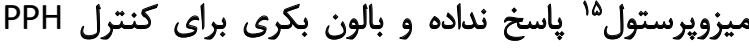

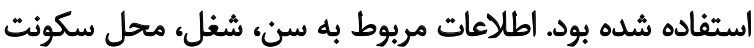

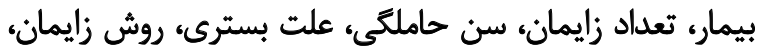

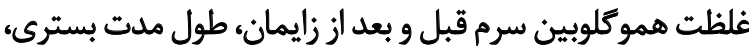

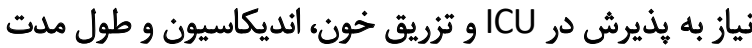

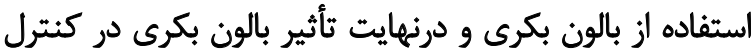

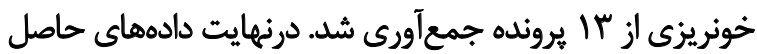

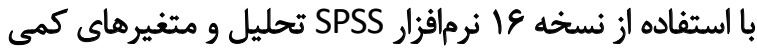

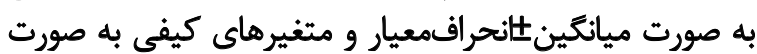
فراوانى(درصد فراوانى) كزارش شدند. در اين مطالعه محققان به كليه اصول و دستورالعملهاى تورئ

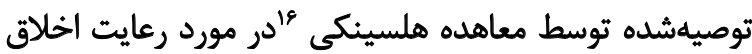

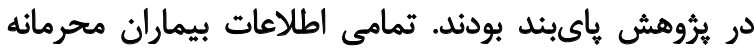

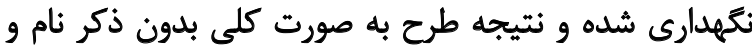

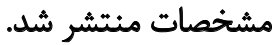

ياقتثهانا

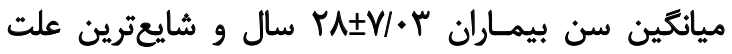

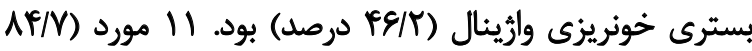

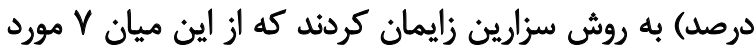

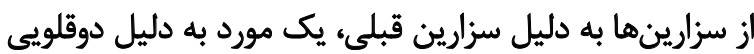

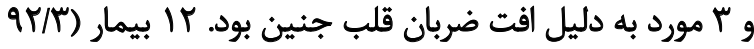

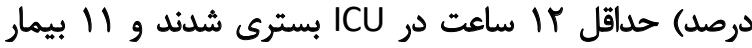

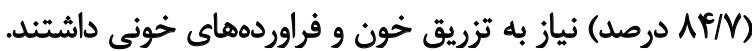

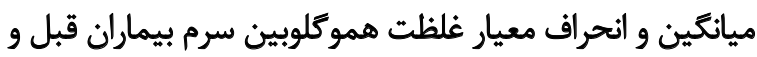

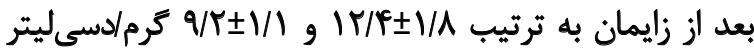

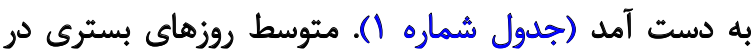

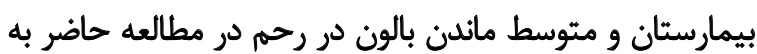

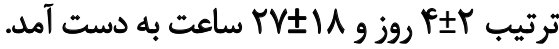

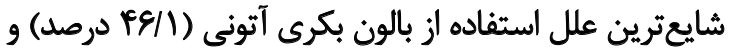

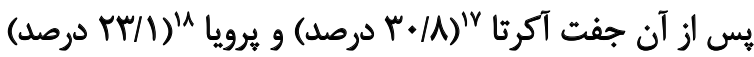

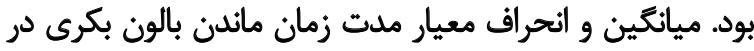

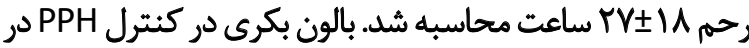

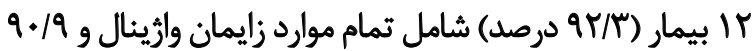

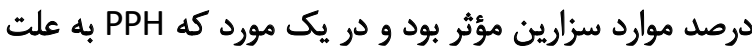

\section{Methylergonovine}

15. Misoprostol

16. Helsinki

17. Placenta accreta

18. Placenta percreta

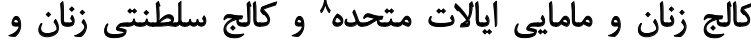

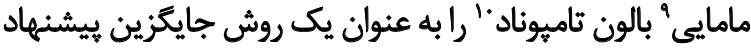

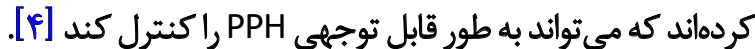

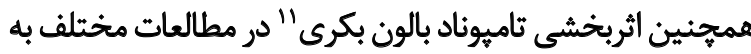

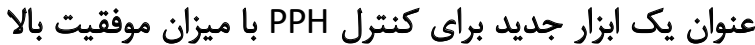

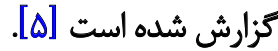

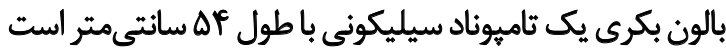

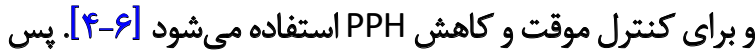

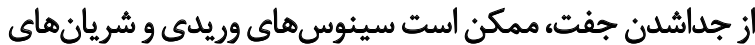

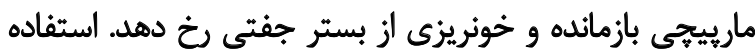

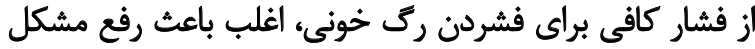

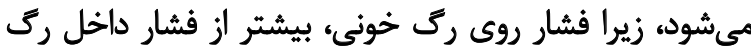

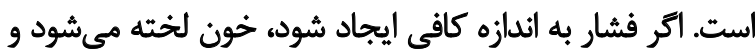

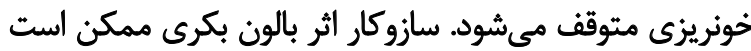

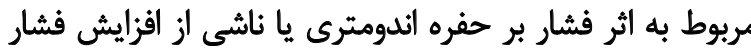

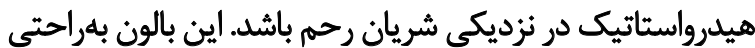

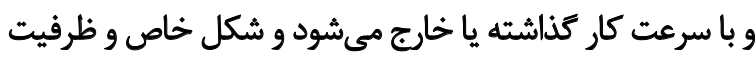

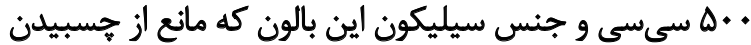

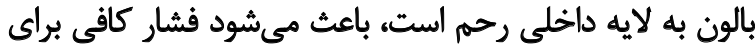

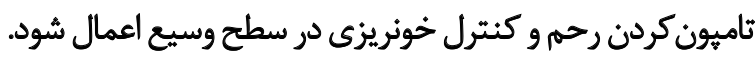

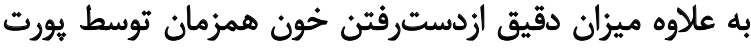

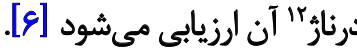

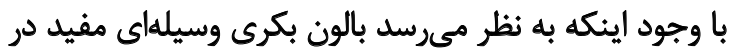

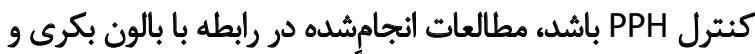

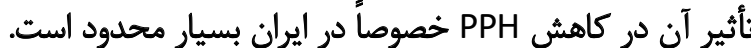

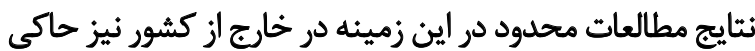

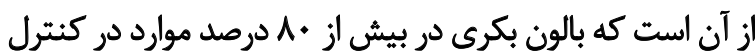

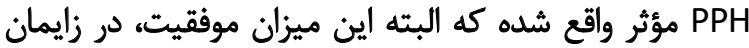

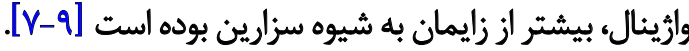
بنابراين زمان تشخيص خونريزى، وجود امكانات مناسب و و

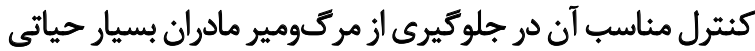

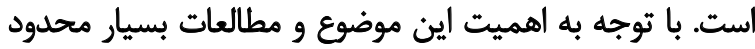

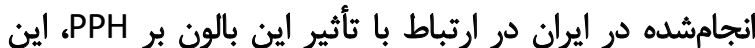

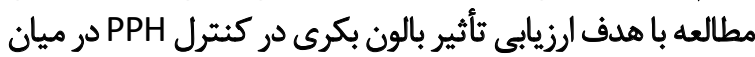
بيماران بسترى در بيمارستان كوثر قزوين صورت كرئ ترفت

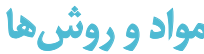

اين مطالعه به روش بررسى موارد" از مهر وqجا تا اسفند

8. American college of obstetricians and gynecologists (ACOG)

9. Royal college of obstetricians and gynaecologists (RCOG)

10. Bballoon tamponade

11. Bakri balloon

12. Drainage port

13. Case series 
جدول (. اطلاعات زميئهاي بيماران مورد مطالعه

\begin{tabular}{|c|c|c|}
\hline فراوانى(درصد) / ميانكين土 اتحراف معيار & \multicolumn{2}{|c|}{ اطلاعات زمينهاى بيماران } \\
\hline$I r(q r / T)$ & خائهدار & \multirow{3}{*}{ شغل } \\
\hline & & \\
\hline$I(Y / M)$ & شاغل & \\
\hline$r(\mid \Delta / Y)$ & شهر & \multirow{2}{*}{ محل سكونت } \\
\hline$\|(A+/ M)$ & روستا & \\
\hline$r(M T / U)$ & نولىيار & \multirow{2}{*}{ تعلداد زايمان } \\
\hline $1 \cdot(v e / 9)$ & مولتعيار & \\
\hline$r(1 \Delta / r)$ & وارئنال & \multirow{2}{*}{ 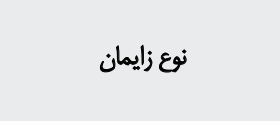 } \\
\hline$\|(A * / M)$ & سزالرين & \\
\hline$r N \pm V /+r$ & & سن بيهار (سال) \\
\hline$r V / r \pm r \Delta / \Delta T$ & & سن حاملكى (هفته) \\
\hline$r \pm r$ & & ملت بسترى (روز) \\
\hline$I F / P \pm 1 / A$ & قبل الز زايمان & \multirow{2}{*}{ هموكلوبين سرم (كرم/دسىليتر) } \\
\hline$q / \pi \pm M$ & بعد از زايمان & \\
\hline
\end{tabular}

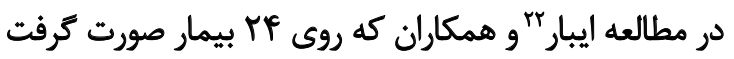

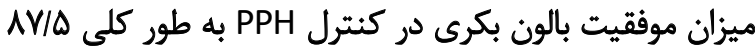

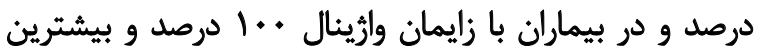

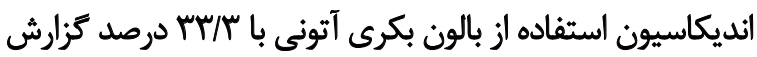

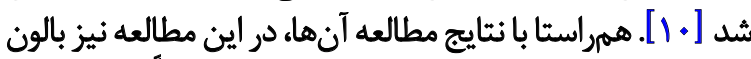

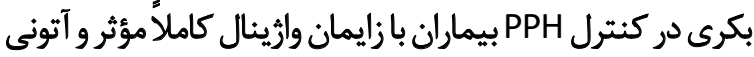

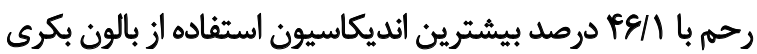

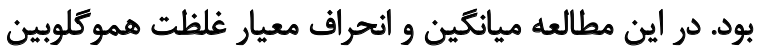

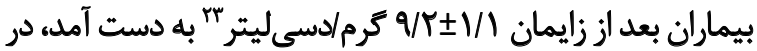

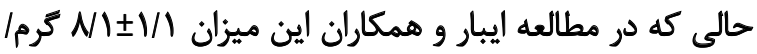

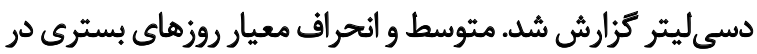

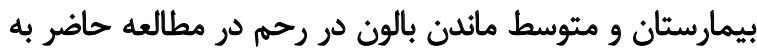

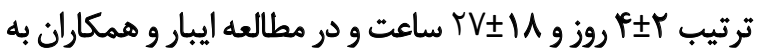

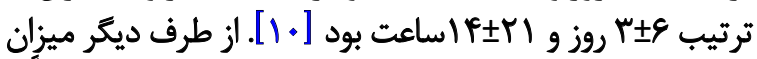

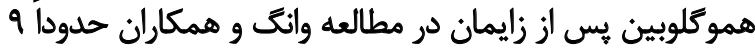

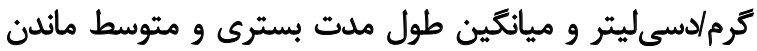

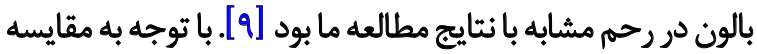

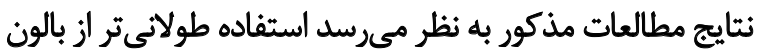

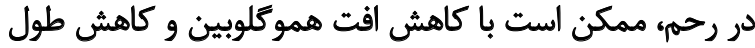

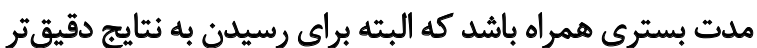

22. Aibar

23. Gram/deci liter
هيرويا به روشهاى مديكال و استفاده از بالون بكرى باسخ نداد، هيستر كتومي انجام شد.

\section{Sydout gon}

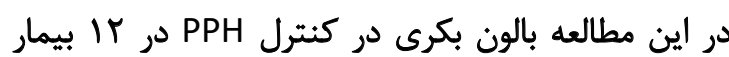

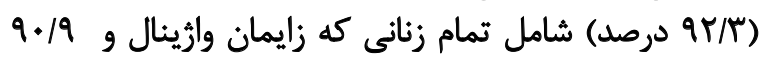

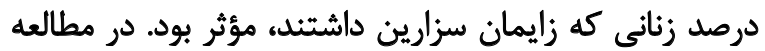

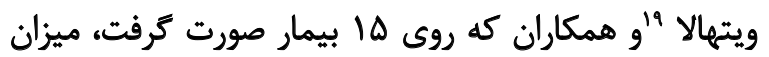

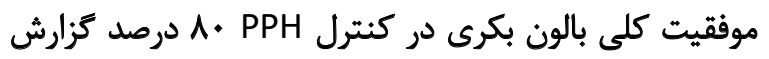

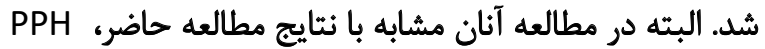

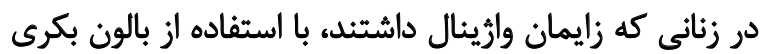

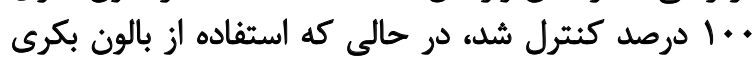

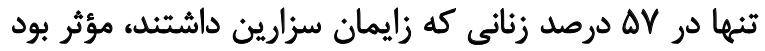

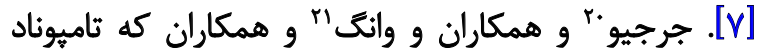

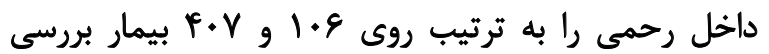

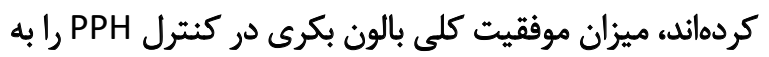

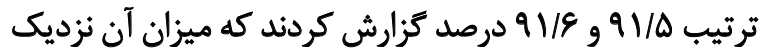

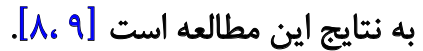




$$
\text { نياز به طراحى مطالعات با حجم نمونه بيشتر است. }
$$

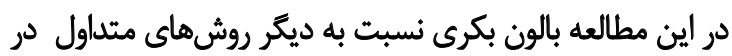

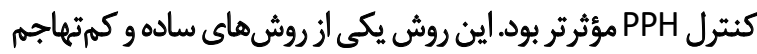

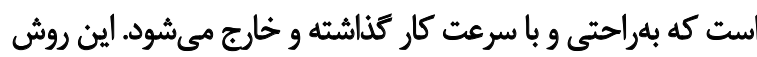

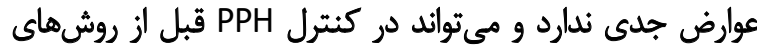

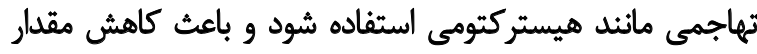

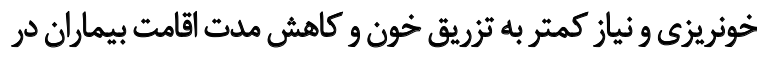

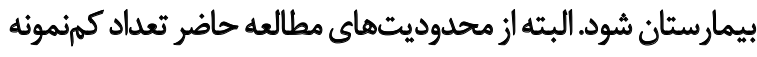

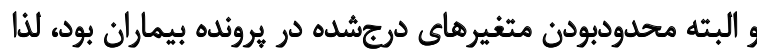

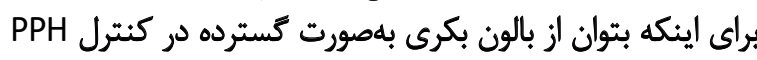

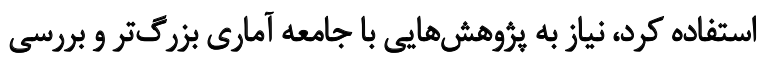

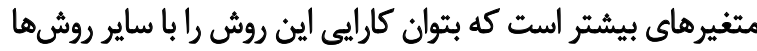

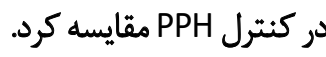

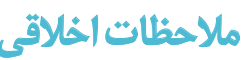

\section{بيروى ازٔ اصول اخلاق ئوهش}

اين مطالعه با كد IR.QUMS.REC.1397.051 از كميته

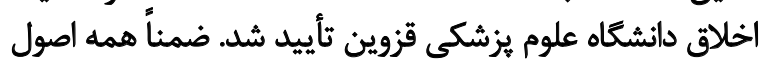

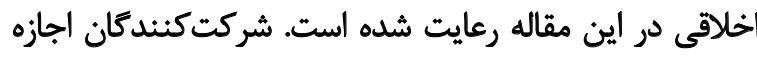

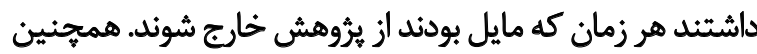

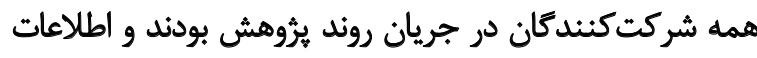

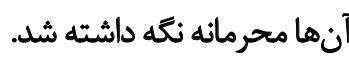

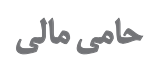

اين تحقيق هيج كمك مالى خاصى از سازمانهاى تأمين مالى داري

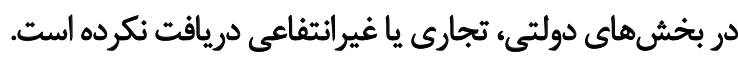

$$
\text { مشاركت نويسندكان }
$$

طراحى مطالعه، جمع آورى دادهها و نكارش نسخه اوليه: مريمه

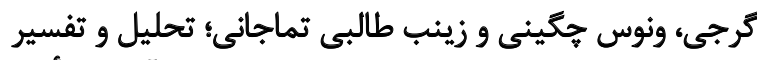

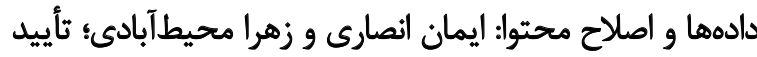

$$
\text { نسخه نهايی: تمام نويسندكان. }
$$

$$
\text { تعارض مثافع }
$$

بنابر اظهار نويسندكان، اين مقاله تعارض منافع ندارد.

$$
\text { سياسكُرأرى }
$$

از همكارى صميمانه آثاى دكتر ملكى، رياست محترم مركز آموزشى

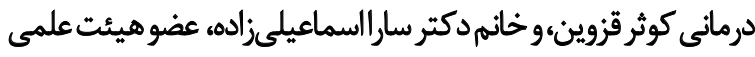

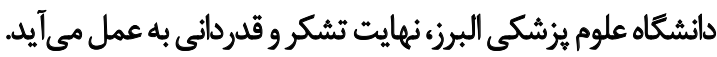




\section{References}

[1] Newsome J, Martin JG, Bercu Z, Shah J, Shekhani H, Peters G. Postpartum hemorrhage. Tech Vasc Interv Radiol. 2017; 20(4):266-73. [DOI:10.1053/j.tvir.2017.10.007] [PMID]

[2] Pakniat H, Chegini V, Shojaei A, Khezri MB, Ansari I. Comparison of the effect of intravenous tranexamic acid and sublingual misoprostol on reducing bleeding after cesarean section: A double-blind randomized clinical trial. J Obstet Gynecol India. 2019; 69(3):239-45. [DOI:10.1007/s13224-018-1181-x] [PMID]

[3] Cekmez Y, Ozkaya E, Öcal FD, Küçüközkan T. Experience with different techniques for the management of postpartum hemorrhage due to uterine atony: Compression sutures, artery ligation and Bakri balloon. Ir J Med Sci. 2015; 184(2):399-402. [DOI:10.1007/s11845-014-1130-3] [PMID]

[4] Darwish AM, Abdallah MM, Shaaban OM, Ali MK, Khalaf M, Sabra AMA. Bakri balloon versus condom-loaded Foley's catheter for treatment of atonic postpartum hemorrhage secondary to vaginal delivery: A randomized controlled trial. J Matern Fetal Neonatal Med. 2018; 31(6):747-53. [DOI:10.1080/14767 058.2017.1297407] [PMID]

[5] Soyama $H$, Miyamoto $M$, Sasa $H$, Ishibashi $H$, Yoshida $M$, Nakatsuka $\mathrm{M}$, et al. Effect of routine rapid insertion of Bakri balloon tamponade on reducing hemorrhage from placenta previa during and after cesarean section. Arch Gynecol Obstet. 2017; 296(3):469-74. [DOI:10.1007/s00404-017-4446-1] [PMID]

[6] Kaya B, Tuten A, Daglar K, Misirlioglu M, Polat M, Yildirim Y, et al. Balloon tamponade for the management of postpartum uterine hemorrhage. J Perinat Med. 2014; 42(6):745-53. [DOI:10.1515/jpm-2013-0336] [PMID]

[7] Vitthala S, Tsoumpou I, Anjum ZK, Aziz NA. Use of bakri balloon in post-partum haemorrhage: A series of 15 cases. Aust $\mathrm{N}$ Z J Obstet Gynaecol. 2009; 49(2):191-4. [DOI:10.1111/j.1479828X.2009.00968.x] [PMID]

[8] Georgiou C. Balloon tamponade in the management of postpartum haemorrhage: A review. Int J Obstet Gynecol. 2009; 116(6):748-57. [DOI:10.1111/j.1471-0528.2009.02113.x] [PMID]

[9] Wang D, Xu S, Qiu X, Zhu C, Li Z, Wang Z, et al. Early usage of Bakri postpartum balloon in the management of postpartum hemorrhage: A large prospective, observational multicenter clinical study in South China. J Perinat Med. 2018; 46(6):64956. [DOI:10.1515/jpm-2017-0249] [PMID]

[10] Aibar L, Aguilar MT, Puertas A, Valverde M. Bakri balloon for the management of postpartum hemorrhage. Acta Obstet Gynecol Scand. 2013; 92(4):465-7. [DOI:10.1111/j.16000412.2012.01497.x] [PMID] 